\title{
PHOTOGRAPHS OF CORONAL STREAMERS \\ FROM A ROCKET ON MAY 9, 1967*
}

\author{
R. Tousey, G. D. Sandlin, and M. J. Koomen** \\ (E. O. Hulburt Center for Space Reșearch, U.S. Naval Research Laboratory, \\ Washington, D.C., U.S.A.)
}

\begin{abstract}
A photograph of the white-light corona from a rocket on May 9, 1967 showed many streamers, all straight and nearly radial, extending across the field of view, 3 to $9 R_{\odot}$. At the North there were three spectacular streamers, making angles with the polar axis of $21^{\circ} \mathrm{E}, 20^{\circ} \mathrm{W}$ and $27^{\circ} \mathrm{N}$. Projected inward, they were radial and passed through the probable positions of the principal plages on the back side.
\end{abstract}

The most recent flight of the U.S. Naval Research Laboratory's white-light coronagraphs took place on May 9, 1967. Two externally occulted, small Lyot coronagraphs were built into the instrument compartment of the biaxial pointing-control section of an Aerobee-150 rocket. The instrument and some earlier results have been described by Tousey (1965), Purcell et al. (1967), and Koomen et al. (1967). In the present note attention is directed to the straight character of the streamers and to the possible origins of the three which appear to come from regions not far from the North Pole.

Simultaneous photographs from the two instruments are reproduced in Figure 1, as unretouched, positive prints. The dark central region is the shadow cast by the external occulter; the dark sectors, in the NW of one and in the SE of the other, are the shadows of the arms supporting each occulter. At the very center of one a white disk has been introduced to show the location and size of the Sun. The other instrument became slightly misaligned during launch, which accounts for the bright crescent at the edge of the occulter's shadow. The decrease in brightness of the corona and streamers with increasing $R$ was compensated by the vignetting action of the external occulter. The useful field covered the range 3 to $9 R_{\odot}$ in the plane perpendicular to the line of sight.

A few artifacts are present, caused by specks on a lens; and the short, bright streak in the NW of the one photograph is the trail of a sunlit particle just beyond the occulter. Mercury is seen almost out of the field in the West, and drawn into a short arc by the precession-caused rotation of the instrument around the solar vector.

* Supported by the National Aeronautics and Space Administration.

** Presented by R. Tousey. 


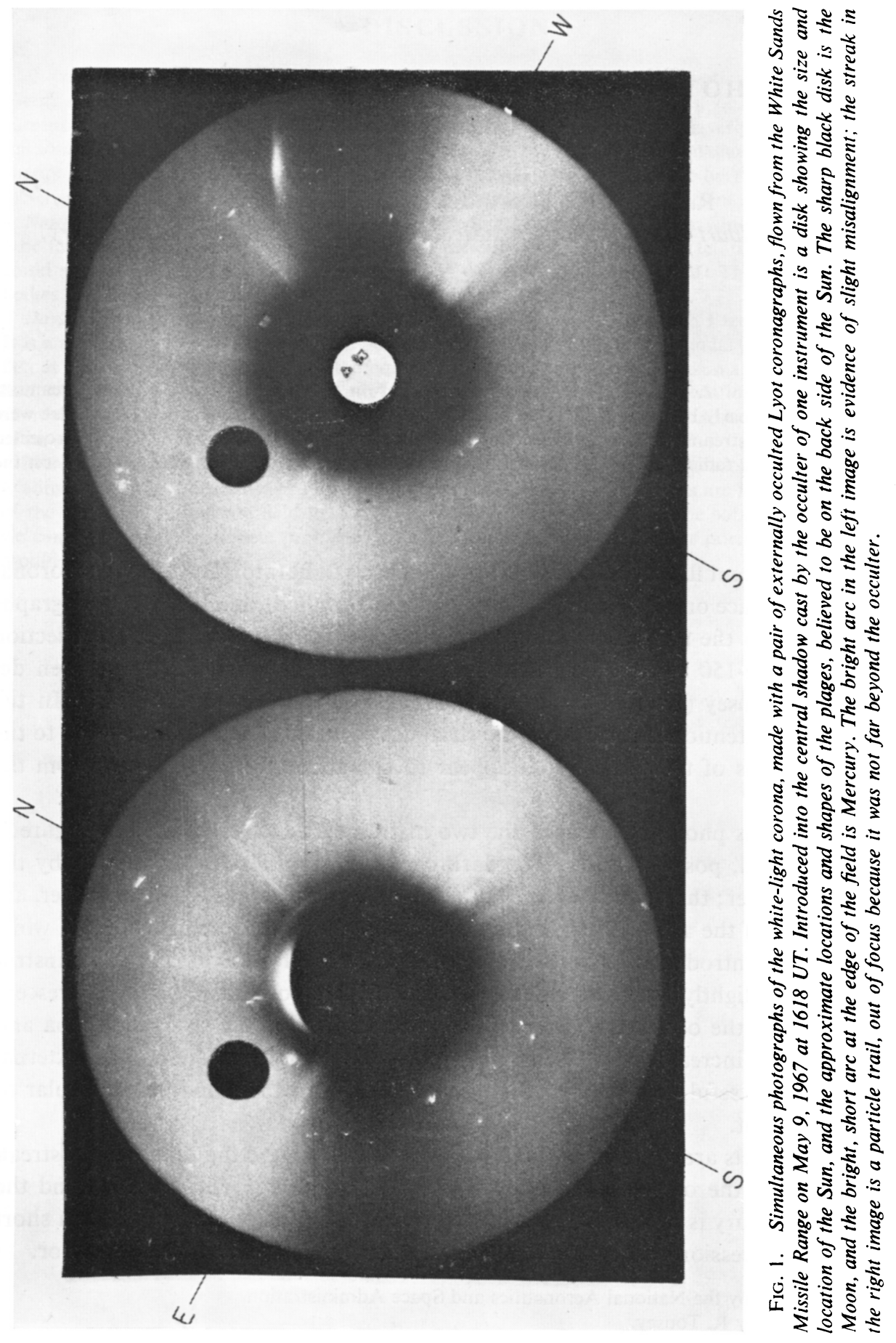


The small, black disk East of the Sun is the Moon. The rocket was launched intentionally on May 9 at 1618 UT in order to have the Moon in the field of view. This aspect of the program has been described by Koomen et al. (1967). The black character of the shadow of the occulting disk and of the Moon shows that the instrumental stray-light level was negligible. The Moon's features are clearly visible in earthshine on the original film.

There are many conspicuous coronal streamers, especially at low solar latitudes. All of the streamers are straight; therefore, the range beyond $3 R_{\odot}$ is largely outside the region where the corona is controlled by complex magnetic fields. It will be difficult to locate the origins of the low-latitude streamers because of the great amount of activity and because of the overlapping and merging of the streamers.

The streamers at high North latitudes can be studied in more detail. The NE streamer is single and appears to form a $21^{\circ}$ angle with the solar axis; the NW streamer is double with angles of $20^{\circ}$ and $27^{\circ}$ to the axis. It appears unlikely that they really originated at high latitudes, because there were no large prominences in suitable positions and because sunspots and intense active centers were not found at those latitudes. They could very well have come from low-latitude centres of activity on the front or back side and thus be seen in projection. On the front side, however, there were no features from which the streamers conceivably could have arisen.

An investigation was made to determine whether or not intense active regions on the back side might have served as origins for these streamers. Examination of the $\mathrm{Ca}-\mathrm{K}$ spectroheliograms for April 25 and May 23, 14 days earlier and later than May 9, showed plages in favorable locations, at latitudes where the solar rotation period is about 28 days. These regions were very large and intense, containing large spots at coordinates $31^{\circ} \mathrm{E}, 26^{\circ} \mathrm{N}$ under plage \# 8785 , at $19^{\circ} \mathrm{E}, 35^{\circ} \mathrm{N}$ under plage \# 8793 which first appeared on April 28 , and at $22^{\circ} \mathrm{W}, 23^{\circ} \mathrm{N}$ under plage \# 8778 . On May 24 the same regions persisted on the Sun, remaining very intense and at roughly the same positions but having grown markedly in area; the Eastern region, designated \# 8818 in this rotation, covered about 8500 millionths of the hemisphere, and the Western region covered about 2500 millionths of the hemisphere. The Eastern region at $247^{\circ}$ longitude was the most active region on the Sun in May, and was the site of the proton-flare events in the last week of May. Average positions for these regions were taken and transferred to the back of the Sun. Their locations and approximate forms are indicated on the white disk in Figure 1 as a view seen through the Sun.

A calculation was made of the apparent angle between each streamer and the solar axis, using the assumed positions of the active centers, making the assumption that the streamers were radial, and taking into account the tilt angle of the Sun and the perspective. Table 1 gives the results.

These values are in very good agreement, in consideration of the errors of measurement and of the uncertainties involved in estimating the centers of the plages.

The conclusion is that these streamers came from active centers that were $50^{\circ}$ to $53^{\circ}$ 


\section{Table 1}

\section{Apparent angle between streamer and solar North}

$\begin{array}{cccc}\text { Plage \#/Streamer } & 8778 / \mathrm{NE} & 8785 / \mathrm{NW} & 8793 / \mathrm{NW} \\ & & & \\ \text { Observed } & 21^{\circ} & 27^{\circ} & 20^{\circ} \\ \text { Calculated } & 20^{\circ} & 29^{\circ} & 17^{\circ}\end{array}$

heliocentric angle behind the limb, and that they were radial. Because of perspective, they were really recorded to about $15 R_{\odot}$. Although they were favorably located to show any twisting, there is no indication of a 'garden hose' effect because they are straight, and project inward along radii passing through the large centers of activity present on the rear side.

\section{Acknowledgements}

We are greatly indebted to Dr. Helen Dodson-Prince, and Miss E. Ruth Hedeman of the McMath-Hulbert Observatory for making available their solar data and for their discussions of the histories of the active regions.

\section{References}

Koomen, M.J., Tousey, R., Seal, R.T.Jr. (1967)

Purcell, J.D., Tousey, R., Koomen, M.J. (1967)

Tousey, R. (1965) in Space Research, VIII. in Space Research, VIII. 